\title{
Evaluation of dyspnea of unknown etiology in HIV patients with cardiopulmonary exercise testing and cardiovascular magnetic resonance imaging
}

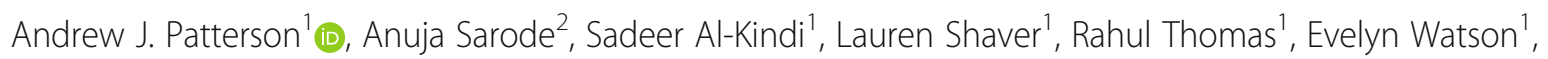
Mohamad Amer Alaiti ${ }^{1}$, Yuchi Liư ${ }^{3}$, Jessie Hamilton ${ }^{3}$, Nicole Seiberlich³, Imran Rashid ${ }^{1}$, Robert Gilkeson ${ }^{4}$, Robert Schilz ${ }^{5}$, Brian Hoit ${ }^{1}$, Trevor Jenkins ${ }^{1}$, Melissa Zullo², Eduardo Bossone ${ }^{6}$, Christopher Longenecker ${ }^{1}$, Orlando Simonetti ${ }^{7}$ and Sanjay Rajagopalan ${ }^{1,4^{*}}$

\begin{abstract}
Aim: Human Immunodeficiency Virus (HIV) patients commonly experience dyspnea for which an immediate cause may not be always apparent. In this prospective cohort study of HIV patients with exercise limitation, we use cardiopulmonary exercise testing (CPET) coupled with exercise cardiovascular magnetic resonance (CMR) to elucidate etiologies of dyspnea.
\end{abstract}

Methods and results: Thirty-four HIV patients on antiretroviral therapy with dyspnea and exercise limitation (49.7 years, $65 \%$ male, mean absolute CD4 count 700) underwent comprehensive evaluation with combined rest and maximal exercise treadmill CMR and CPET. The overall mean oxygen consumption $\left(\mathrm{VO}_{2}\right)$ peak was reduced at $23.2 \pm 6.9 \mathrm{ml} / \mathrm{kg} / \mathrm{min}$ with 20 patients (58.8\% of overall cohort) achieving a respiratory exchange ratio $>1$. The ventilatory efficiency (VE) $\mathrm{NCO}_{2}$ slope was elevated at $36 \pm 7.92$, while ventilatory reserve (VE: maximal voluntary ventilation (MW)) was within normal limits. The mean absolute right ventricular (RV) and left ventricular (LV) contractile reserves were preserved at $9.0 \% \pm 11.2$ and $9.4 \% \pm 9.4$, respectively. The average resting and postexercise mean average pulmonary artery velocities were $12.2 \pm 3.9 \mathrm{~cm} / \mathrm{s}$ and $18.9 \pm 8.3$ respectively, which suggested lack of exercise induced pulmonary artery hypertension (PAH). LV but not RV delayed enhancement were identified in five patients. Correlation analysis found no relationship between peak $\mathrm{VO}_{2}$ measures of contractile RV or LV reserve, but LV and RV stroke volume correlated with $\mathrm{PET} \mathrm{CO}_{2}(p=0.02, p=0.03)$.

Conclusion: Well treated patients with HIV appear to have conserved RV and LV function, contractile reserve and no evidence of exercise induced PAH. However, we found evidence of impaired ventilation suggesting a noncardiopulmonary etiology for dyspnea.

Keywords: HIV, CPET, Exercise CMR, Contractile Reserve

\footnotetext{
* Correspondence: Sanjay.Rajagopalan@UHhospitals.org

'Harrington Heart and Vascular Institute, University Hospitals, Cleveland, $\mathrm{OH}$

USA

${ }^{4}$ University Hospitals Cleveland Medical Center, Department of Radiology,

Cleveland, OH, USA

Full list of author information is available at the end of the article
}

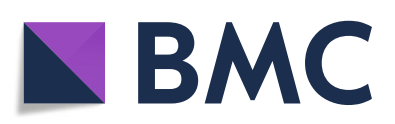

(c) The Author(s). 2020 Open Access This article is licensed under a Creative Commons Attribution 4.0 International License, which permits use, sharing, adaptation, distribution and reproduction in any medium or format, as long as you give appropriate credit to the original author(s) and the source, provide a link to the Creative Commons licence, and indicate if changes were made. The images or other third party material in this article are included in the article's Creative Commons licence, unless indicated otherwise in a credit line to the material. If material is not included in the article's Creative Commons licence and your intended use is not permitted by statutory regulation or exceeds the permitted use, you will need to obtain permission directly from the copyright holder. To view a copy of this licence, visit http://creativecommons.org/licenses/by/4.0/ The Creative Commons Public Domain Dedication waiver (http://creativecommons.org/publicdomain/zero/1.0/) applies to the data made available in this article, unless otherwise stated in a credit line to the data. 


\section{Introduction}

Unexplained dyspnea in patients with human immunodeficiency virus (HIV) can be multifactorial and may portend a poor prognosis especially with diagnoses such as HIV pulmonary arterial (PA) hypertension (PAH) [13]. Indeed, the earliest signs of eventual PAH are impaired right ventricular (RV) contractile reserve and a phase of exercise induced PAH [4] [5]. The presence of these features may help identify subsets of patients with propensity for developing resting $\mathrm{PAH}$ and/or RV contractile dysfunction for preemptive therapy [6]. While the gold standard for evaluation of cardiopulmonary performance and assessment of PA pressure remains invasive right heart catheterization in combination with exercise, the use of such an approach for comprehensive screening is fairly involved $[7,8]$. Other tools such as stress echocardiography to evaluate for changes in tricuspid valve velocities, increase in E/e' and contractile dysfunction are often used, but are limited by imaging windows that could be suboptimal in many patients, particularly with exercise [8]. Coupling cardiovascular magnetic resonance (CMR) with cardiopulmonary exercise testing (CPET) may allow comprehensive noninvasive functional and structural analysis of patients with dyspnea of unclear etiology and could provide valuable prognostic and diagnostic information [9]. In this prospective study, we evaluated HIV patients with subjective exercise limitation, with no other obvious evidence of cardiopulmonary disease to comprehensively assess ventricular contractile reserve, PA velocities and cardiopulmonary ventilatory indices using a treadmill exercise CMR imaging protocol combined with CPET.

\section{Methods}

Patient selection

A total of 34 patients were enrolled out of 53 eligible patients with HIV that were referred for functional assessment between May 2017 and January 2019 (Fig. 1). All

\section{EXHALTED CONSORT Diagram}

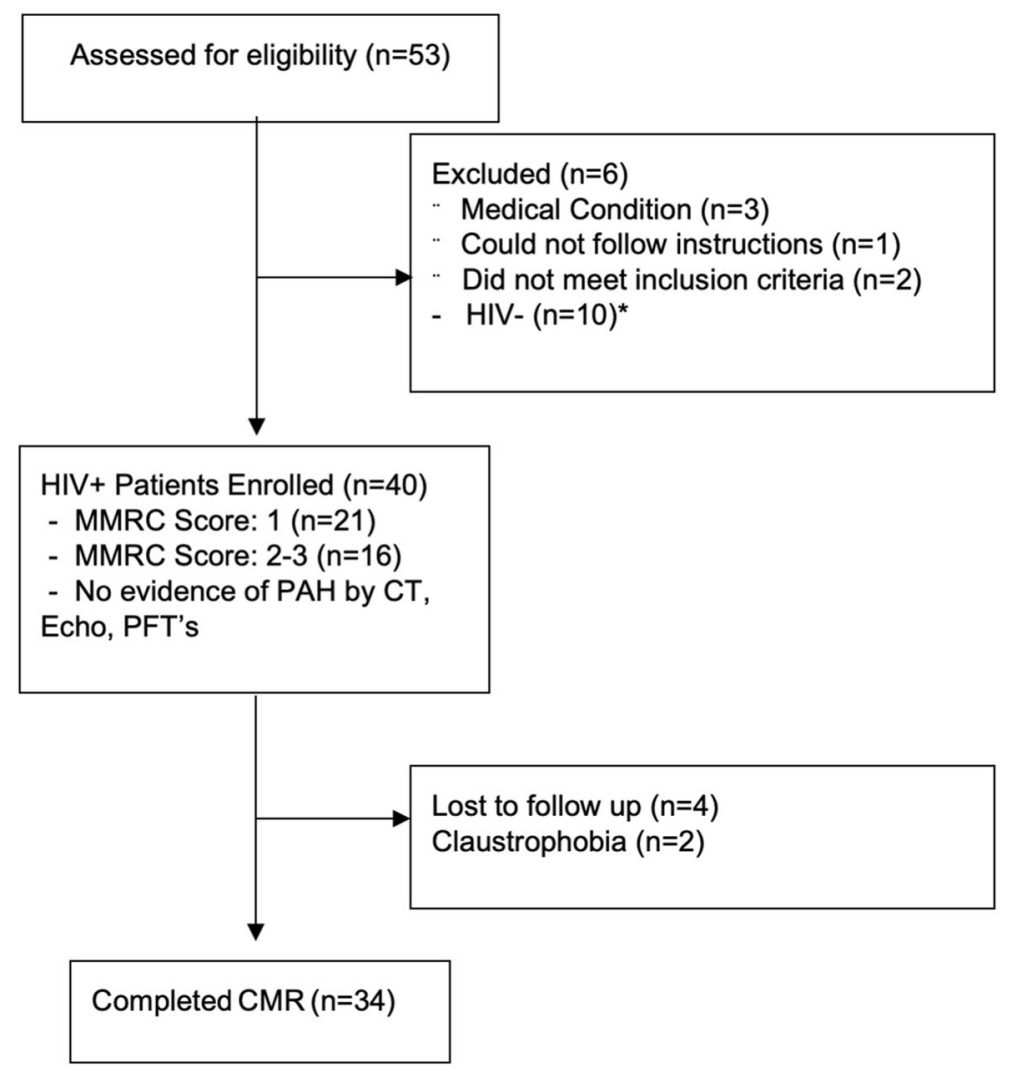

*HIV- patients were enrolled, but not included in these analyses

Fig. 1 Diagram of selected patients that underwent combined CPET/CMR for evaluation of dyspnea. A total of 53 enrolled of which 34 completed screening and underwent testing. CPET - cardiopulmonary exercise testing, CT - computed tomography, CMR - cardiovascular magnetic resonance, HIV - human immunodeficiency virus, MMRC - Modified Medical Research Council, PFTs - pulmonary function testing 
patients signed a consent form and the study was conducted in accordance with IRB standards of University Hospitals Cleveland Medical Center and in accordance with Helsinki convention.

The inclusion criteria included adults aged 18-65 years with confirmed HIV, with unexplained persistent or progressively worsened dyspnea for at least 6 weeks, and were described previously [10]. Screening echocardiography with a cutoff of resting PA systolic pressures of $\leq 40 \mathrm{mmHg}$ using peak tricuspid regurgitation velocities coupled with right atrial pressures, pulmonary function testing to rule out obstructive and restrictive pulmonary disease, computed tomography $(\mathrm{CT})$ screening if indicated to exclude parenchymal pulmonary disease as well as evidence of thromboembolic disease with main PA size $(<3 \mathrm{~cm})$ were used to assess for eligibility. Patients were required to have exercise limitation based on the Modified Medical Research Council (MMRC) dyspnea scale, the most commonly used validated subjective scale to assess shortness of breath in patients with respiratory disease as previously described [11]. The scale uses a 0 4 scale with 0 indicating shortness of breath only with strenous exercise. 1-3 are graded progressive symptoms. Four indicates the subject is too short of breath to leave their house or they have shortness of breath when dressing.
Exclusion criteria included contraindications to CMR (e.g., metallic implants, severe claustrophobia); acute coronary syndrome, transient ischemic attack, stroke or critical limb ischemia during the last 6 months or coronary/peripheral revascularization within the last 3 months; concurrent potentially life threatening arrhythmia or symptomatic arrhythmia; documented left ventricular (LV) ejection fraction (LVEF) $<45 \%$; evidence of pulmonary infection in the past 4 weeks; Evidence of another etiology of individual's dyspnea such as reactive airway disease, heart failure, intrinsic lung disease, and decompensated cirrhosis; chronic kidney disease with estimated glomerular filtration rate (eGFR) of $<40 \mathrm{ml} /$ $\min / 1.73 \mathrm{~m}^{2}$ or recent acute kidney injury; hemoglobin $<8 \mathrm{~g} / \mathrm{dL}$; platelets $<50,000 / \mathrm{mm}$; pregnancy; active drug use, either by history or urine toxicology screen.

\section{Stress protocol}

Subjects were scheduled for combined CPET and exercise CMR (Central Illustration: Fig. 2) as previously described [10]. Pre-exercise images were reviewed by an experienced cardiologist and/or radiologist prior to exercise. After the completion of pre-exercise images, patients were transferred to a CMR-compatible treadmill to perform a symptom limited CPET (Parvo Medics, Sandy, Utah, USA) using a standardized protocol $[9,10]$.

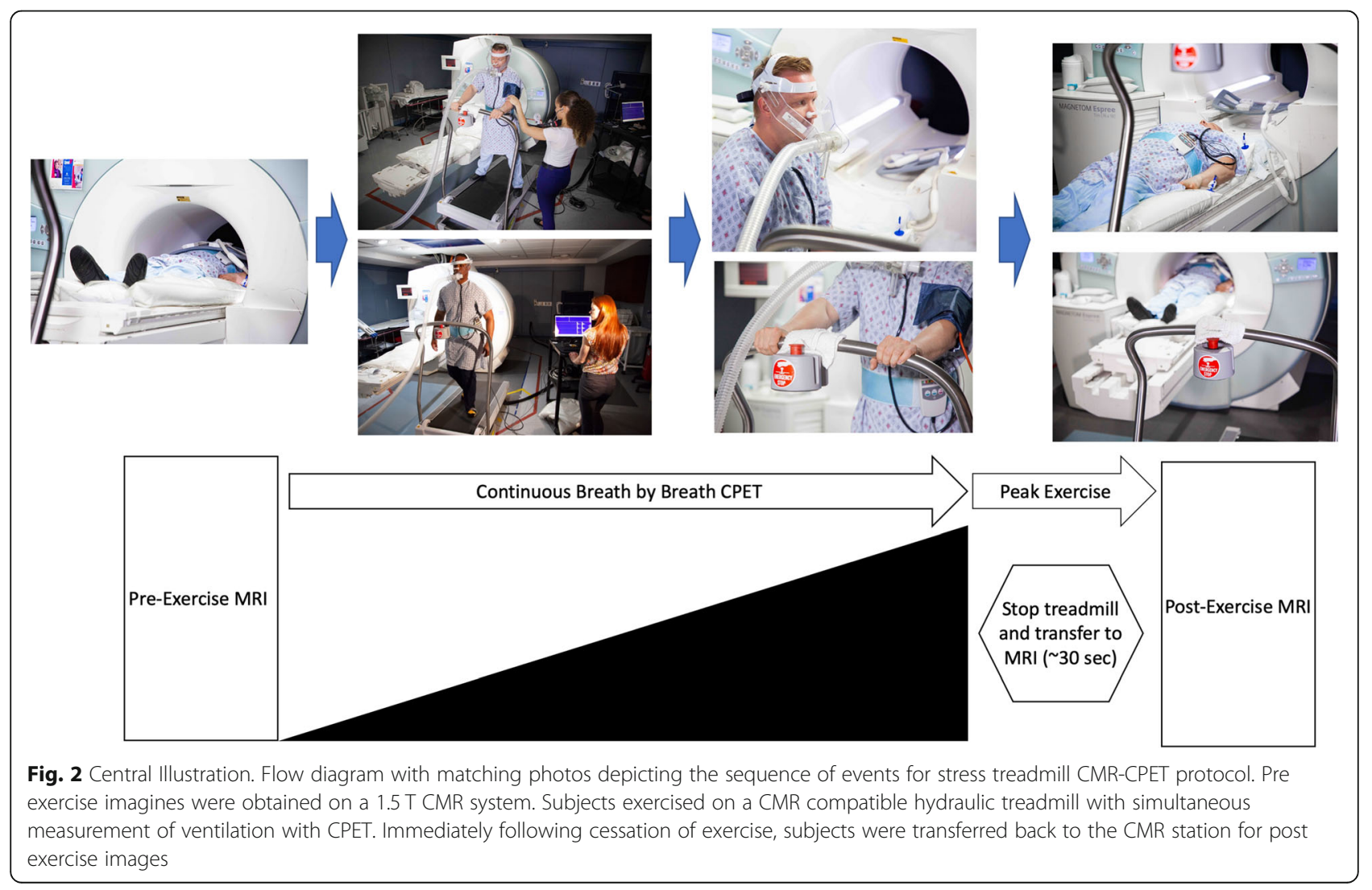


The heart rate was monitored continuously, with blood pressure and rating of perceived exertion (RPE) obtained at end of each stage. All participants were encouraged to exercise to exhaustion, which was defined as a respiratory exchange ratio (RER) $\geq 1.0$. A 10 -s averaged sampling interval was used to measure baseline and peak exercise $\mathrm{PetCO}_{2}$, ventilatory efficiency $\left(\mathrm{VE} / \mathrm{VCO}_{2}\right)$, ventilatory threshold (VT), and oxygen consumption $\left(\mathrm{VO}_{2}\right)$ values. VT was determined by the v-slope method [12] and peak $\mathrm{VO}_{2}(\mathrm{ml} / \mathrm{kg} / \mathrm{min})$ was defined as the maximum oxygen uptake during the last $60 \mathrm{~s}$ leading up to exercise termination. Heart rate and blood pressure were monitored continuously throughout the post-exercise scan. Arteriovenous oxygen $\left(\mathrm{A}-\mathrm{VO}_{2}\right)$ difference was calculated as previously described [13].

\section{Cardiovascular magnetic resonance imaging}

CMR was performed on a $1.5 \mathrm{~T}$ (Espree ${ }^{\mathrm{T} \omega}$, Siemens Healthineers, Erlangen, Germany) using a 32-channel phased array coil and a standard clinical protocol for structural, functional, and phase-contrast breath-hold imaging at rest was followed according to published international guidelines [14]. A real-time, through-plane velocity encoding sequence based on gradient-echo echo-planar imaging (GRE-EPI) with Shared Velocity Encoding (SVE) reconstruction was used for evaluation of PA velocities [15]. An echo train length of 7 and linear $\mathrm{k}$-space acquisition order resulted in an echo time (TE) of $5 \mathrm{~ms}$ and a repetition time (TR) of $10 \mathrm{~ms}$ at a velocity encoding (VENC) of $150 \mathrm{~cm} / \mathrm{s}$. Four shots per image were used to collect a total of $28 \mathrm{k}$-space lines resulting in an acquisition time of $40 \mathrm{~ms}$ for each full kspace dataset $[2405 \mathrm{~Hz} /$ pixel readout bandwidth, $84 \times$ 128 pixel reconstructed matrix, $10 \mathrm{~mm}$ slice, $268 \mathrm{~mm} \times$ $350 \mathrm{~mm}$ rectangular field-of-view $(3.2 \mathrm{~mm} \times 2.7 \mathrm{~mm}$ pixels)]. A $25^{\circ}$ rapid water-excitation pulse was used to suppress fat, and Maxwell correction was used to account for the effect of concomitant gradients on the phase maps. Parallel imaging technique TGRAPPA with acceleration rate 3 was used to reconstruct 84 lines per image. Three orthogonal slices across the PA were prescribed with the highest velocity vector within these slices and the time velocity integral calculated. We used a non-triggered real-time balanced steady-state free precession (bSSFP) acquisition using a radial scheme for $\mathrm{k}$ space acquisition. We chose this method for improved post exercise image quality and reduction of scan time. A total of 60 accelerated short-axis images were acquired for each slice in order to ensure capture of a complete cardiac cycle (approximately $2.5 \mathrm{~s}$ of imaging per slice) which allowed for adequate discrimination of ectopic beats. Heart IT software (Heart Imaging Technologies, Durham, North Carolina, USA) allows for manual selection of end-systole and end-diastole for each slice excluding papillary muscles allowing an aggregate compilation of volumes for quantitative ejection fraction. This use of real time cine acquisitions has been utilized previously and shown to have great correlation with standard cine acquisition with less artifact [16]. A stack of real time cine images in the short-axis plane with slice thickness of $8 \mathrm{~mm}$ (2-mm inter-slice gap) were acquired fully covering both ventricles from base to apex. For real time cine bSSFP the following parameters were used: TE $0.98 \mathrm{~ms}$; TR $86.26 \mathrm{~ms}$, temporal resolution $46 \mathrm{~ms}, \alpha=65^{\circ}$, band width $1488 \mathrm{~Hz} /$ pixel. PA velocities, mean and peak gradients were measured at rest and stress See Fig. 3a-e for examples of rest and stress ventricular volumes and pulmonary velocity encoding. Contractile reserve was determined by assessing the change in ejection fraction at peak exercise.

The assessment of RV and LV fibrosis were obtained using late gadolinium enhancement (LGE) imaging 10 min after administration of multihance gadolinium agent $(0.15 \mathrm{mmol} / \mathrm{kg})$. Short axis images were acquired using a single shot breath hold phase contrast inversion recovery technique of the entire myocardium. Images were assessed using a semi-quantitive method with HeartIT (Precession) imaging software. This method uses the American Heart Association 17 segment model where six segments for both basal and mid portions, four segments for apical and one segment for apex are scored. Each segment can yield a minimum of 0 for normal myocardium, 1 for $0-25 \%, 2$ for $25-50 \%, 3$ for $50-75 \%$ and a maximum of 4 for transmural $75-100 \%$ LGE/scar. The sum of the score was used to derive estimated percentage of myocardium with late gadolinium enhancement.

\section{Statistical analysis}

Statistical analysis was performed using SAS (Version 9.4, SAS Institute, Cary, North Carolina, USA). Categorical data were expressed as percentage while continuous variables were reported as mean \pm SD along with $95 \%$ CI. Two-sided dependent group $t$-test $(\alpha=0.05)$ was used to compare the differences between pre and post exercise CMR and CPET observations. To summarize the relationship between $\triangle \mathrm{CMR}$ parameters and $\triangle$ CPET parameters univariate analysis was performed using simple linear regression. Predictors of RVand LV reserve were assessed using stepwise multiple linear regression. $P$ values of $<0.05$ were considered significant. Bland altman analysis was done in 10 subjects for right and left ventricle ejection fraction to assess for observer variability.

\section{Results}

Figure 2 shows subjects undergoing exercise treadmill testing with CPET and CMR analysis. For test subjects, the mean age of the participates was $49.7 \pm 11.0$ years and the mean body mass index (BMI) was $28.8 \pm 7.6 \mathrm{~kg} / \mathrm{m}^{2}$. The 


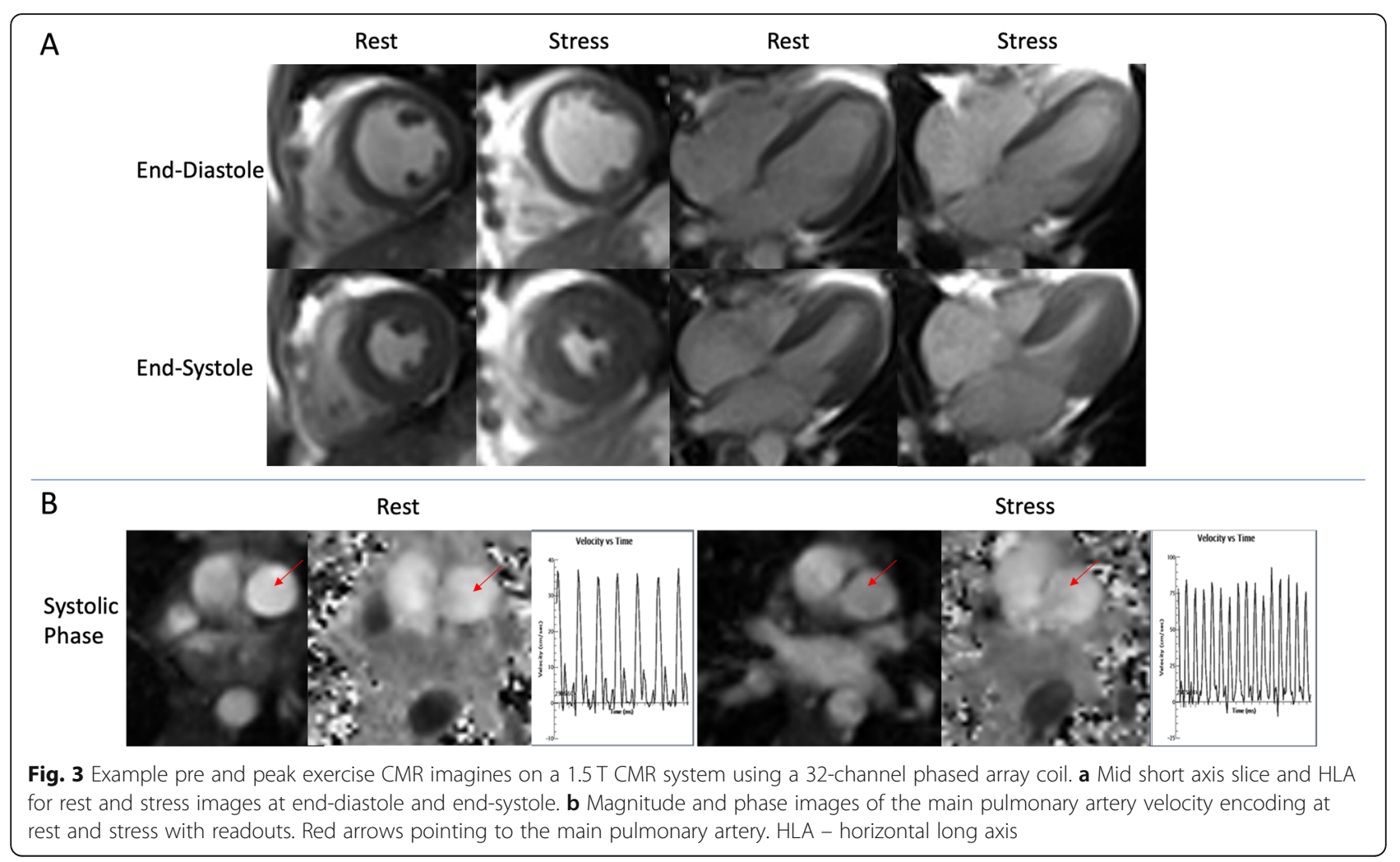

mean duration of HIV exposure was 23.9 years. Table 1 enumerates the baseline characteristics of study cohort, while Table 2 and Table 3 details the specifics of exercise testing. The mean percent of age predicted maximal heart rate was $90.7 \pm 17.9 \mathrm{bpm}$ and mean metabolic equivalents (MET)s achieved was $9.6 \pm 3.9$. The time to transfer the patient, prior to commencing stress imaging, averaged < $35 \mathrm{~s}$, which is within acceptable thresholds advocated by the American Heart Association [17]. During initial screening, pulmonary functional testing (PFTs) was used to assess baseline function. The mean forced expiratory volume in $1 \mathrm{~s}\left(\mathrm{FEV}_{1}\right)$ was $2.9 \pm 8.6 \mathrm{~L}$ and a mean \% predicted $\mathrm{FEV}_{1}$ of $91.9 \pm 18.4$ and mean $\% \mathrm{FEV}_{1}$ / forced vital capacity (FVC) was $92.1 \pm 12.0$. Maximal voluntary ventilation (MVV), a surrogate for maximal ventilatory capacity, was calculated as $\mathrm{FEV}_{1} \times 40$ to measure ventilatory reserve. For the present cohort, the mean MVV was 116 and the mean VE (Max) 67.5 \pm 29.2 . The ventilatory reserve (VE: MVV) is a measurement of the intrinsic pulmonary limitations to exercise [7] with normative range $<0.7$. We found a VE: MVV of 0.6. The pre-exercise imaging was slightly variable, given need for optimization of the phase contrast imaging planes to ensure adequate visualization of the PA and prescription of orthogonal planes for assessment of PA velocities. Figures 4, 5 and Table 4 details the peak exercise hemodynamic parameters. Notably, mean peak $\mathrm{VO}_{2}$ was decreased at $23.2 \pm 6.9 \mathrm{ml} / \mathrm{kg} / \mathrm{min}$ and mean $\mathrm{VE} /$
$\mathrm{VCO}_{2}$ slope was increased at $35.8 \pm 7.9$ and overall with 20 patients ( $58.8 \%$ of overall cohort) achieving a RER $>1$ indicating a maximal exercise effort.

\section{Contractile reserve}

To assess contractile reserve, LVEF and RV ejection fraction (RVEF) and stroke volumes were quantified at rest and stress. Basal ventricular function ejection fraction for RV and LV were within normal limits (Fig. 4, Table 4). Using an absolute increase of $5 \%$ as a measure of contractile reserve [4], both RV and LV augmented appropriately with exercise (Fig. 4, Table 4). The mean absolute contractile reserve for RV was $9.0 \% \pm 11.2$ and $\mathrm{LV}$ was $9.4 \% \pm 9.4$. The mean change in RV and LV stroke volume was $7.2 \pm 25.3$ $\mathrm{ml}$ and $8.6 \pm 19.7 \mathrm{ml}$, respectively. We measured peak and average PA velocities as well as mean and peak gradients for rest and stress to evaluate for evidence for PAH. We have previously shown exceptional correlation between PA velocities, namely average PA velocity with invasively measured mean PA pressures and calculated pulmonary vascular resistance (PVR) with right heart catheterization at rest in PAH patients [18]. Elevated mean PA pressures correlated with a reduced average PA velocity as derived from CMR. The mean average PA velocity for rest and stress was $12.2 \pm 3.9$ and $18.9 \pm 8.3$ (See Fig. 5, Table 4) indicating the absence of exercise-induced PAH. The mean and peak 
Table 1 Demographic characteristics of the EXHALTED population $(n=34)$

\begin{tabular}{|c|c|c|c|c|}
\hline & & Total $n$ & Mean \pm SD/Frequency (\%) & $95 \% \mathrm{Cl}$ \\
\hline \multirow[t]{5}{*}{ Demographics } & Age, years & 34 & $49.68 \pm 11.01$ & $(45.84-53.52)$ \\
\hline & African American & 34 & $28(82.35)$ & $(68.85-95.85)$ \\
\hline & Unemployed & 34 & $26(76.47)$ & $(61.45-91.49)$ \\
\hline & Current smokers & 34 & $20(58.82)$ & $(41.39-76.25)$ \\
\hline & Current alcohol use, yes & 34 & $19(55.88)$ & $(38.30-73.47)$ \\
\hline \multirow[t]{6}{*}{ Labs } & Absolute CD4 count & 34 & $699.62 \pm 416.81$ & $(554.18-845.05)$ \\
\hline & Absolute CD8 count & 34 & $769.59 \pm 307.47$ & $(662.31-876.87)$ \\
\hline & White Blood Cell count, Thousand/uL & 33 & $5.72 \pm 1.97$ & $(5.02-6.42)$ \\
\hline & Hemoglobin, g/dl & 33 & $13.59 \pm 1.61$ & $(13.02-14.17)$ \\
\hline & Creatinine, mg/dL & 33 & $1.02 \pm 0.25$ & $(0.93-1.11)$ \\
\hline & Albumin, g/dL & 33 & $4.19 \pm 0.29$ & $(4.09-4.29)$ \\
\hline \multirow[t]{6}{*}{ Medication Use } & Aspirin & 34 & $9(26.47)$ & $(10.85-42.10)$ \\
\hline & $\beta$-Blockers & 34 & $3(8.82)$ & $(0.00-18.87)$ \\
\hline & Statins & 34 & $9(26.47)$ & $(10.85-42.10)$ \\
\hline & Diuretics & 34 & $2(5.88)$ & $(0.00-14.22)$ \\
\hline & Calcium Channel Blockers & 34 & $6(17.65)$ & $(4.15-31.15)$ \\
\hline & ACE Inhibitors/ARB & 34 & $9(26.47)$ & $(10.85-42.10)$ \\
\hline \multirow[t]{8}{*}{ Medical History } & Pulmonary Hypertension, no & 33 & $33(100.00)$ & $(100.00-100.00)$ \\
\hline & Cancer Diagnosis, no & 34 & $30(88.24)$ & (76.82-99.65) \\
\hline & Hypertension, no & 34 & $20(58.82)$ & $(41.39-76.25)$ \\
\hline & Diabetes, no & 34 & $28(82.35)$ & $(68.85-95.85)$ \\
\hline & Hyperlipidemia, no & 34 & $25(73.53)$ & $(57.90-89.15)$ \\
\hline & Coronary artery disease, no & 34 & $33(97.06)$ & $(91.08-100.00)$ \\
\hline & History of stroke, no & 34 & $32(94.12)$ & (85.78-100.00) \\
\hline & Chronic kidney disease, no & 34 & $34(100.00)$ & $(100.00-100.00)$ \\
\hline
\end{tabular}

gradients did not change significantly with exercise and remained within normal limits (Data not shown).

\section{Metabolic gas exchange relationships}

We further assessed the relationship between functional changes in LVEF, RVEF and peak PA velocity relative to gas exchange parameters. Correlation matrix analysis between change in $\mathrm{VO}_{2}, \mathrm{VE} / \mathrm{VCO}_{2}$, $\mathrm{PetCO}_{2}$ relative to the change in RVEF, stroke volume and PA velocities (Supplemental Table 1). The change in LVEF, RVEF, peak or average PA velocity did not account for changes in $\mathrm{VO}_{2}$, peak $\mathrm{PetCO}_{2}$ and VE/ $\mathrm{VO}_{2}$. Interestingly, a relationship was observed between change in $\mathrm{PetCO}_{2}$ in response to exercise and change in LV and RV stroke volume (Supplemental table 1).

\section{Assessment of skeletal muscle strength}

Prior to exercise testing, patients baseline skeletal muscle strength was assessed by hand grip (Lafayette Instrument,
Lafayette, Indiana, USA) and quadricep (Hoggan Scientific, LLC, Salt Lake City, Utah, USA) strength. Participants were graded on a scale of $0-5$. Scores were averaged over 3 trials. We did not find significant evidence of muscle weakness in hand and quadriceps testing as all patients scored greater than 4 with testing.

\section{Assessment of LV and RV fibrosis}

The presence of scar/fibrosis portends a poor prognosis in many cardiomyopathies. To assess for scar/fibrosis, we performed LGE imaging using single shot breath hold inversion recovery sequences in addition to stress imaging protocols. The presence of RV enhancement was not identified; however enhancement was seen in the LV in five (15\%) of 34 patients with average scar burden of $5.6 \%$. Predominant location of scar was found in the mid myocardium or junctional RV insertion site. Subendocardial or transmural scar indicative of prior infarct was not observed. 
Table 2 Exercise CMR Testing Times

\begin{tabular}{llll}
\hline & Mean \pm SD & Min & Max \\
\hline Exercise Time (min:sec) & $08: 17 \pm 03: 43$ & $01: 21$ & $00: 20$ \\
Time Exercise End to Start Images (min:sec) & $00: 34 \pm 00: 22$ & \\
Pre-exercise Scan (min)* & $20-25$ & \\
Post-exercise scan (min)* & $15-20$ & \\
\hline
\end{tabular}

For consistency, CMR images were read by two experienced cardiologists for RV and LV volumetric analysis as well as average velocities. We used random effects model to evaluate inter-rater reliability [19]. Our results show appropriate reliability for RV volumetric analysis with ICC $=0.73$ and LV volumetric analysis with $\mathrm{ICC}=0.82$. Additionally, we found agreement for average PA velocity at rest with $\mathrm{ICC}=0.70$ and stress $\mathrm{ICC}=0.72$. See supplemental figure $1 \& 2$.

\section{Discussion}

In the present study, we sought to evaluate causes of dyspnea in a cohort of well treated HIV patients with exercise limitation and no overt signs of PAH using a comprehensive combined CPET/CMR platform that would allow unprecedented ability to simultaneously assess for multiple central and peripheral etiologies of dyspnea [7]. Overt evidence of lung disease and preexisting cardiac dysfunction and resting pulmonary hypertension were excluded through screening procedures such as chest CT, echocardiogram and PFT's. Parenchymal lung disease, abnormalities in resting ventilatory reserve and reactive airway disease were excluded. VE:MVV, a measurement of the intrinsic pulmonary limitation to exercise [7] was within normal limits $(<0.7)$, with mean VE:MVV at 0.6 , which made intrinsic lung pathology less likely. The feasibility of combined exercise CPET and CMR has been previously noted by other groups for investigation of cardiopulmonary pathology $[13,20]$. The juxtaposition of these technologies greatly simplifies the assessment of the patient with unexplained dyspnea and facilitates diagnosis through an iterative process of exclusion. We ensured that patients were exercised to their peak capacity, with transfers occurring rapidly, ensuring that imaging was performed within suggested parameters for near peak exercise imaging of the ventricles [17].

We initially identified substantially impaired maximal exercise capacity $\left(\mathrm{VO}_{2}\right.$ peak and $\left.\mathrm{VCO}_{2}\right)$ in this HIV patient population with mild subjective dyspnea. This is well described and has been noted by us and others [21, 22]. Given the impairment of peak $\mathrm{VO}_{2}$ capacity, we systemically interrogated for cardiac causes of dyspnea. We considered exercise-induced increase in pulmonary vascular resistance, given that this may present with dyspnea and considered an early marker for resting PAH [8, 23, 24]. This diagnosis could be very easily ascertained through assessment of pulmonary artery velocities with real-time phase contrast CMR assessment of PA velocities at peak exercise. The mean average PA velocities in this population at rest and with exercise corresponded to a normal range of mean PA pressures, using previously described regression equations that have correlated mean average PA velocities with invasive right heart catheterization in patients with PAH [18]. We were able to image the PA reproducibly to obtain a reliable estimate for PA velocities at peak exercise in all patients.

Poor contractile reserve may also help explain dyspnea in patients with otherwise structurally normal resting ventricles but has not been adequately evaluated in HIV patients with dyspnea. The presence of contractile

Table 3 Resting and peak exercise heart and blood pressure

\begin{tabular}{|c|c|c|c|}
\hline & Mean \pm SD & Min & Max \\
\hline \multicolumn{4}{|l|}{ Resting } \\
\hline Heart Rate (bpm) & $75.25 \pm 14.73$ & 58.00 & 122.00 \\
\hline Supine Systolic Blood Pressure (mmHg) & $122.71 \pm 20.22$ & 96.00 & 173.00 \\
\hline Supine Diastolic Blood Pressure (mmHg) & $81.04 \pm 12.48$ & 54.00 & 103.00 \\
\hline \multicolumn{4}{|l|}{ Peak Exercise } \\
\hline Heart Rate (bpm) & $155.67 \pm 36.29$ & 115.00 & 196.00 \\
\hline$\%$ Age Predicted Max Heart Rate & $90.7 \pm 17.86$ & 67.00 & 149.00 \\
\hline Systolic Blood Pressure (mmHg) & $161.37 \pm 20.26$ & 118.00 & 190.00 \\
\hline Diastolic Blood Pressure (mmHg) & $84.37 \pm 15.26$ & 52.00 & 118.00 \\
\hline METS Achieved & $9.63 \pm 3.89$ & 4.2 & 16.50 \\
\hline
\end{tabular}


reserve in patients with nonischemic cardiomyopathy, irrespective of stressors and imaging modality is associated with $80 \%$ reduction in mortality and hospitalization [4]. RV contractile reserve is particularly important in patients with the diagnosis of $\mathrm{PAH}$, where inadequate ventricular response to exercise may predict eventual development of fixed PAH and mortality $[6,8]$. We did not find evidence of compromised biventricular contractile reserve. We then turned our attention to myocardial scar/fibrotic burden as early indications of dysfunction and found only a minority of subjects (5 patients) could be identified with LGE which primarily involved the LV and not the RV. There are reports of higher predisposition for fibrosis in HIV patients compared to age match control populations [25]. In patients with HIV, LGE at the RV insertion site, inferolateral wall or septum has been previously noted and thought to be prevalent and related to prior myocarditis [26, 27]. However, we did not consistently observe LGE, suggesting a lack of RV/LV remodeling to fully explain symptomology. We measured diastolic indices in the LV and RV at rest and with exercise and did not observe any changes in diastolic function excluding a contribution of diastolic dysfunction (data not shown).
In the setting of mean RER 1.0, we observed reduced peak $\mathrm{VO}_{2}(23.2 \mathrm{ml} / \mathrm{kg} / \mathrm{min})$ and elevated peak $\mathrm{VE} / \mathrm{VCO}_{2}$ (mean 35.8). The mean $\mathrm{PetCO}_{2}$ at VT was also reduced at $27 \mathrm{mmHg}$. Lewis et al., demonstrated in patients with heart failure with reduced ejection fraction, $\mathrm{VE} / \mathrm{VCO}_{2}$ slope is negatively correlated to RVEF and significantly associated with pulmonary vascular resistance during exercise, without relationship to LVEF or systemic resistance [28]. These changes were ameliorated by the addition of a selective inhibitor of type 5 phosphodiesterase [28]. In our observations, there was no relationship between $\mathrm{VE} / \mathrm{VCO}_{2}$ slope and resting RVEF or with RV contractile reserve. This may have been related to the fact that our patient population did not have evidence of resting or provoked abnormalities in contractile function. However, the variability of LV and RV stroke volume were directly related to changes in $\mathrm{PetCO}_{2}$ (Supplemental Table 1). Previous studies have found a relationship between $\mathrm{PetCO}_{2}$ values and cardiac output as well as severity of heart failure [29, 30], however the significance of this finding is unclear and may warrant further investigation in future studies. The finding of VE: MVV of $60 \%$, demonstrated a normal breathing reserve, normal PFTs and lack of elevated right sided pressures suggest that a pulmonary etiology is

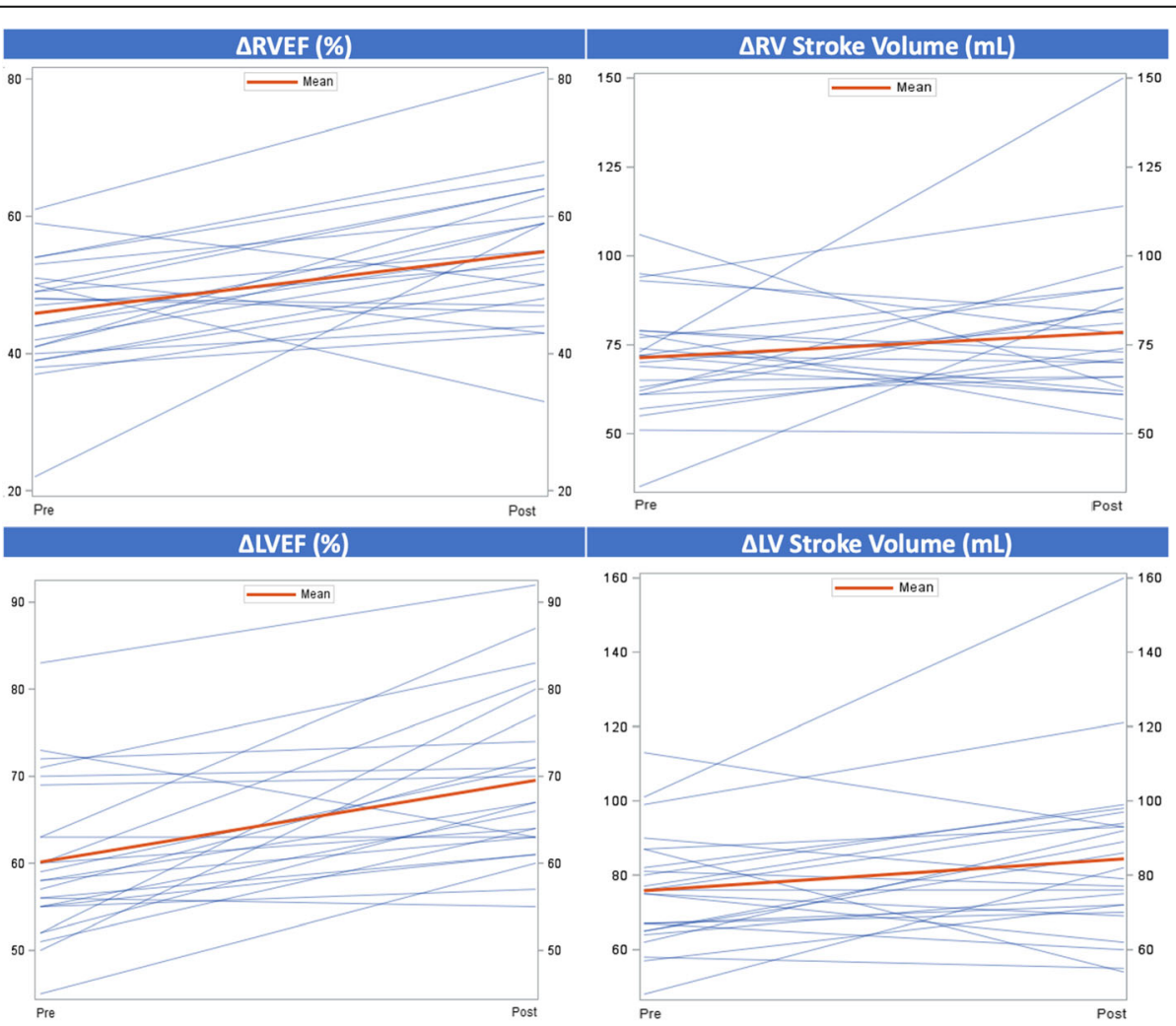

Fig. 4 Assessment of RV (Top Panel) and LV (Bottom Panel) contractile reserve. Scatter plots depicting rest and peak changes in ejection fraction and stroke volume for RV (EF $p=0.001, \mathrm{SV} p=0.08)$ and LV (EF $p<0.001$, SV $p=0.02)$. Each line is representative of an individual patient. Dark red line indicates mean value. $\mathrm{RV}=$ right ventricle, $\mathrm{LV}=$ left ventricle, $\mathrm{EF}=$ ejection fraction, $\mathrm{SV}=$ stroke volume 


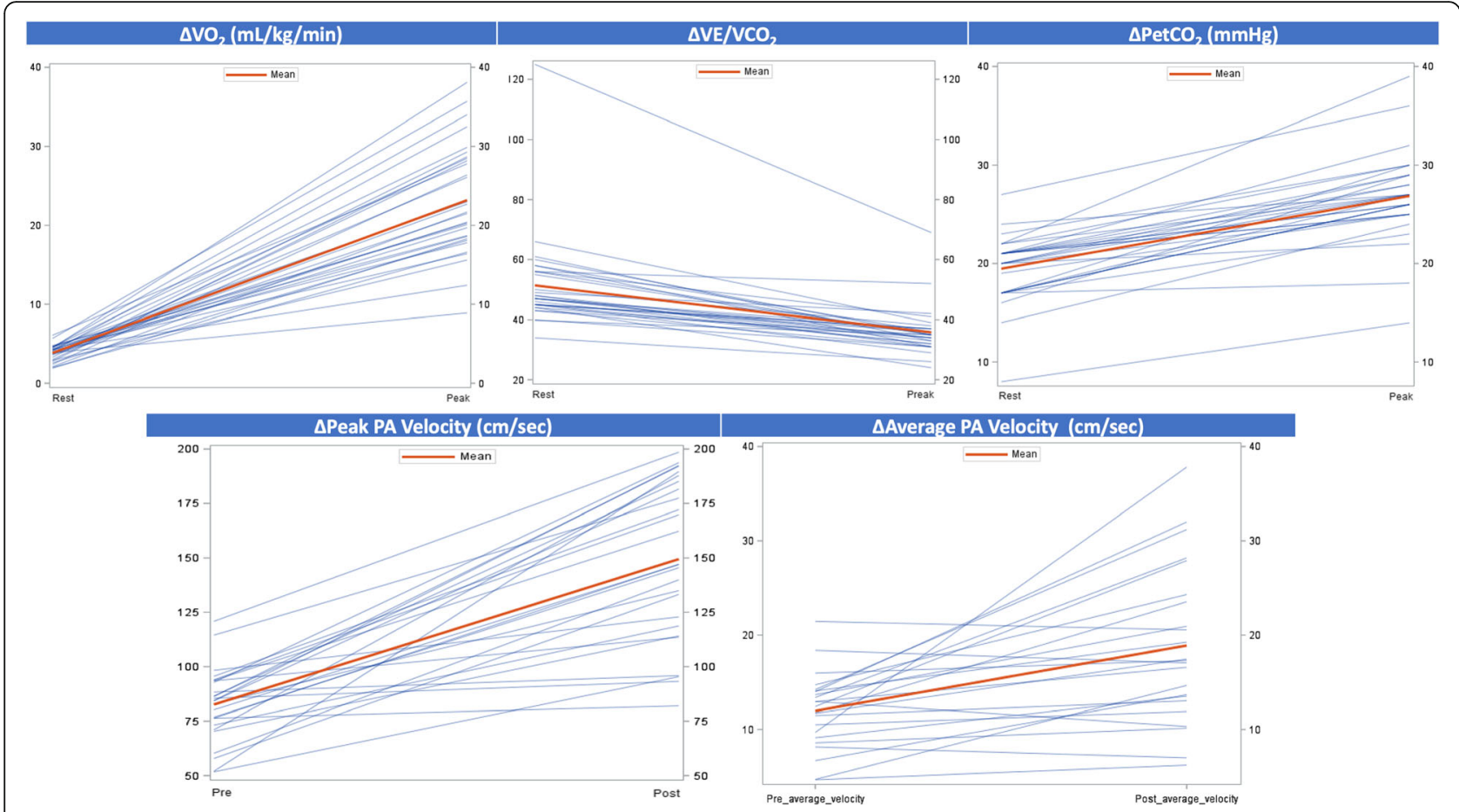

Fig. 5 Assessment of cardiopulmonary function and main pulmonary velocities. Top panel indicates resting cardiopulmonary function as assessed with CPET and values measured occur at peak exercise for $\mathrm{VO}_{2}, \mathrm{VE} / \mathrm{VCO}_{2}$ and $\mathrm{PETCO}$. Lower panel depicts resting and peak average and peak pulmonary velocities as measured through velocity encoding sequence with VENC set points of 100 for rest and 150 with stress. Each line represents an individual patient. Dark red line indicates mean value

unlikely, and that dyspnea may relate to peripheral mechanisms of alterations in $\mathrm{VO}_{2}$ peak.

Skeletal muscle mass is an independent predictor of peak $\mathrm{VO}_{2}$ and $\mathrm{VE} / \mathrm{VCO}_{2}$ slope, which could explain our observations [31]. Peripheral limitations to exertion in people living with HIV can include sarcopenia [32], skeletal myopathy from direct mitochondrial toxicity [26], and prolonged exposure to elevated systemic inflammation [33]. Skeletal myopathy in patients with HIV can occur as a result of direct/prolong exposure to the virus. Additionally, anti-retroviral therapies may cause myopathies through direct mitochondrial toxicity [34]. Although macroscopic muscle strength testing did not show overt evidence of a manifest myopathy, a subclinical process could help explain dyspnea in the patient cohort. The average duration of HIV exposure in our patient population was 23.9 years, which likely indicates prolong exposure to the virus and anti-retroviral therapies. Although majority of patients reached a RER of 1, there were a notable number of patients unable to reach target exertion goals suggesting either deconditioning or symptom limiting fatigue. Indeed, subjective dyspnea may be a reflection of overall deconditioning and may not be related to progression of disease. The average BMI was $29 \mathrm{Kg} / \mathrm{m}^{2}$, suggesting that majority of patients were overweight, if not obese. Indeed, there is a rising epidemic of overweight/obesity amongst HIV patients which will have implications for cardiovascular health [35].

Prior studies have used comparable system with CMR compatible ergometer/treadmills coupled with CPET in pediatric patients with PAH and tetralogy of Fallot [12]. Although similar, our system utilized a CMR compatible hydraulic treadmill with gradual stress protocols [10,17]. The advantage of using a combined CMR/CPET platform is the ability to comprehensively assess cardiopulmonary function with ventilatory function, quantification of RV/LV volumes, velocity encoding of great vessels and scar/fibrosis burden with LGE and evaluation of electrocardiogram findings. Conditions such as low flow/ low gradient aortic stenosis, load dependent mitral valve regurgitation and monitoring of treatment response of patients with $\mathrm{PAH}$ are amongst emerging areas of interest for comprehensive cardiopulmonary evaluation.

\section{Limitation}

A major limitation of this study was that it was conducted in a single center small cohort of HIV patients. The small sample size reflects the challenge in recruiting patients who met the specific study requirements of not having evidence of structural disease or baseline contractile dysfunction but were found to be symptomatic while meeting the additional criteria of undergoing exercise testing in a the CMR environment. We utilized average velocity with CMR derived 
Table 4 CPET and CMR

\begin{tabular}{|c|c|c|c|c|c|c|c|}
\hline & Variable & Mean & SD & Median & Min & Max & IQR \\
\hline \multirow[t]{6}{*}{ CPET } & VO2/kg (VT) (ml/kg/min) & 20.1 & 4.7 & 19.9 & 11.6 & 30.5 & $17.6-23.3$ \\
\hline & VO2/kg (Peak) (ml/kg/min) & 23.20 & 6.88 & 21.70 & 8.90 & 38.10 & $18.3-28.50$ \\
\hline & VE/VCO2 (VT) & 32.5 & 4.3 & 32 & 25 & 41 & $29.5-35.0$ \\
\hline & VE/VCO2 (Peak) & 35.81 & 7.92 & 35.00 & 24.00 & 69.00 & $32.00-37.00$ \\
\hline & PetCO2 (VT) (mmHg) & 27.8 & 6 & 28 & 13 & 37 & $25.0-31.0$ \\
\hline & PetCO2 (Peak) (mmHg) & 26.97 & 4.55 & 27.00 & 14.00 & 39.00 & $25.00-29.00$ \\
\hline \multirow[t]{18}{*}{ CMR } & LV End Diastolic Volume (ml) & 129.59 & 32.86 & 126.00 & 81.00 & 231.00 & $107.00-146.00$ \\
\hline & RV End Diastolic Volume (ml) & 161.56 & 43.30 & 156.00 & 101.00 & 300.00 & $133.00-179.00$ \\
\hline & Pre-LV Stroke Volume (ml) & 75.30 & 15.96 & 75.00 & 48.00 & 113.00 & $64.00-87.00$ \\
\hline & Post-LV Stroke Volume (ml) & 84.38 & 22.67 & 80.50 & 54.00 & 160.00 & $71.00-93.50$ \\
\hline & Pre-RV Stroke Volume (ml) & 70.70 & 16.45 & 72.00 & 35.00 & 106.00 & $61.00-79.00$ \\
\hline & Post-RV Stroke Volume (ml) & 78.54 & 21.20 & 73.50 & 50.00 & 150.00 & $64.50-86.50$ \\
\hline & Pre-LVEF (\%) & 59.67 & 8.60 & 58.00 & 45.00 & 83.00 & $54.00-63.00$ \\
\hline & Post-LVEF (\%) & 69.54 & 9.64 & 67.00 & 55.00 & 92.00 & $63.00-75.50$ \\
\hline & Pre-RVEF (\%) & 45.33 & 8.36 & 47.00 & 22.00 & 61.00 & $39.00-51.00$ \\
\hline & Post-RVEF (\%) & 54.83 & 10.25 & 54.50 & 33.00 & 81.00 & $47.50-61.50$ \\
\hline & RV contractile Reserve & 9.00 & 11.22 & 11.00 & -17.00 & 37.00 & $4.50-14.50$ \\
\hline & LV contractile reserve & 9.42 & 9.39 & 9.00 & -10.00 & -28.00 & $2.00-15.00$ \\
\hline & $\begin{array}{l}\text { Pre-Exercise Peak PA Velocity } \\
\qquad(\mathrm{cm} / \mathrm{sec})\end{array}$ & 82.57 & 16.32 & 84.25 & 51.72 & 120.84 & 73.19-93.48 \\
\hline & $\begin{array}{l}\text { Post-Exercise Peak PA Velocity } \\
\qquad(\mathrm{cm} / \mathrm{sec})\end{array}$ & 149.46 & 36.65 & 147.04 & 82.01 & 198.58 & $118.72-185.10$ \\
\hline & Change in Peak PA Velocity & 66.88 & 35.14 & 69.71 & 5.64 & 137.61 & $43.74-97.34$ \\
\hline & $\begin{array}{c}\text { Pre Exercise Average PA } \\
\text { Velocity }\end{array}$ & 12.19 & 3.92 & 12.68 & 4.66 & 21.45 & $8.63-16.63$ \\
\hline & $\begin{array}{c}\text { Post Exercise Average PA } \\
\text { Velocity }\end{array}$ & 18.88 & 8.34 & 17.37 & 6.22 & 37.8 & $7.15-27.60$ \\
\hline & $\mathrm{A}-\mathrm{VO}_{2}(\mathrm{~mL} \mathrm{02} / 100 \mathrm{~mL}$ blood$)$ & 5.70 & 1.7 & 5.34 & 3.4 & 11.84 & $4.89-6.43$ \\
\hline
\end{tabular}

velocity encoding sequence across the main PA as a surrogate for mean PA pressure. Although previous studies have shown acceptable correlation between average resting PA velocity and invasively measured mean PA velocity it is unknown if the average velocity at peak stress correlates closely with invasively measured mean PA pressure under exercise conditions. An additional limitation of the study is we did not measure peripheral lean muscle mass to screen for sarcopenia as a correlate of submaximal exercise performance.

\section{Conclusion}

Using treadmill stress with CMR/CPET analysis provided comprehensive cardiopulmonary function testing. The data suggests that in well treated patients with HIV, contractile dysfunction with exercise, exercise $\mathrm{PAH}$, diastolic function or presence of the RV/ LV scar are not significant contributors to unexplained dyspnea. Replication of this data in a large multicenter approach may increase the clinical applicability. The etiology of symptoms points to a noncardiopulmonary etiology that may involve subclinical myopathy related to chronic exposure to the HIV virus, drugs and/or physical deconditioning. Future studies may evaluate poorly or uncontrolled retroviral illness in assessing cardiopulmonary function with comprehensive platforms such as CMR/CPET. 


\section{Supplementary information}

Supplementary information accompanies this paper at https://doi.org/10. 1186/s12968-020-00664-6.

Additional file 1 Figure S1 Bland Altman analysis of right ventricular ejection fraction (RVEF) and left ventricular ejection fraction (LVEF) contractile reserve.

Additional file 2: Figure S2 Bland Altman analysis of rest and stress average pulmonary artery velocity as assessed using real time velocity encoding sequence.

Additional file $\mathbf{3}$ Table S1 Pearson Correlation Coefficients

\section{Abbreviations}

A-VO2: Arteriovenous oxygen difference; BMl: Body mass index; bSSFP: Balanced steady state free precession; CMR: Cardiovascular magnetic resonance; CPET: Cardiopulmonary exercise testing; CT: Computed tomography; eGFR: Estimated glomerular filtration rate; EPI: Echo planar imaging; $F_{E V}$ : Forced expiratory volume in one second; FVC: Forced vital capacity; GRE: Gradient echo; HIV: Human immunodeficiency virus; LGE: Late gadolinium enhancement; LV: Left ventricle/left ventricular; LVEF: Left ventricular ejection fraction; METs: Metabolic equivalents; MMRC: Modified Medical Research Council; MW: Maximal voluntary ventilation; PA: Pulmonary artery; PAH: Pulmonary artery hypertension; PFT: Pulmonary function test; PVR: Pulmonary vascular resistance; RER: Respiratory exchange ratio; RPE: Rating of perceived exertion; RV: Right ventricle/right ventricular; RVEF: Right ventricular ejection fraction; SV: Stroke volume; SVE: Shared velocity encoding; TE: Echo time; TR: Repetition time; VE: Ventilatory efficiency; VENC: Velocity encoded; $\mathrm{VO}_{2}$ : Oxygen consumption; $\mathrm{VT}$ : Ventilatory threshold

\section{Acknowledgements}

Not applicable.

\section{Authors' contributions}

$A J^{\ddagger, \delta, \varphi}, \mathrm{AS}^{\delta, \varphi}, \mathrm{SA}^{\neq, \delta}, \mathrm{LS}^{\ddagger, \S, \delta, \varphi}, \mathrm{RT}^{\ddagger}, \mathrm{EW}^{\ddagger}, \mathrm{MA}^{\omega, \neq}, \mathrm{YL}^{*}, J H^{*}, \mathrm{NS}^{*}, \mathrm{IR}^{\omega}, \mathrm{RG}^{\omega}, \mathrm{RS}^{\omega}, \mathrm{BH}^{\omega}$ $\mathrm{T} J^{\delta, \varphi}, \mathrm{MZ}^{\omega, \delta}, \mathrm{EB}^{\delta, \varphi}, \mathrm{CL}^{\omega, \delta, \varphi}, \mathrm{OS}^{\delta, \varphi}, \mathrm{SR}^{\omega, \neq, *, \delta, \varphi}$. $\omega$ Conception and design of project. ‡ Data Acquisition. § Collaboration in the design, implementation and use of the CMR compatible Hydraulic Treadmill in the study population. * CMR sequence design and optimization. SCollaboration in data interpretation and analysis. $\varphi$ Manuscript draft and revision. The author(s) read and approved the final manuscript.

\section{Funding}

This work was supported by the National Institutes of Health (HL 125060) awarded to Dr. Rajagopalan.

\section{Availability of data and materials}

The datasets used and/or analyzed during the current study are available from the corresponding author on reasonable request.

\section{Ethic approval and consent to participate}

All patients signed a consent form and the study was conducted in accordance with IRB standards of University Hospitals Cleveland Medical Center and in accordance with Helsinki convention.

\section{Consent for publication}

All data used or contained in study were consented for publication as stipulated by intuitional IRB policy. In addition, all data is deidentified in compliance with Health Insurance Portability and Accountability Act (HIPAA) standards.

\section{Competing interests}

The authors declare that they have no competing interests.

\section{Author details}

${ }^{1}$ Harrington Heart and Vascular Institute, University Hospitals, Cleveland, $\mathrm{OH}$, USA. ${ }^{2}$ Kent State University, College of Public Health, Kent, OH, USA.

${ }^{3}$ Michigan University, Department of Biomedical Engineering, Ann Arbor, MI, USA. ${ }^{4}$ University Hospitals Cleveland Medical Center, Department of
Radiology, Cleveland, OH, USA. ${ }^{5}$ University Hospitals Cleveland Medical Center, Department of Pulmonology, Cleveland, OH, USA. ${ }^{6}$ Cardiology Division, Cardarelli Hospital, Naples, Italy. ${ }^{7}$ Ohio State University Department of Cardiovascular Medicine, Columbus, OH, USA.

Received: 20 November 2019 Accepted: 25 August 2020

Published online: 12 October 2020

\section{References}

1. Barnett CF, Hsue PY. Human immunodeficiency virus-associated pulmonary arterial hypertension. Clin Chest Med. 2013;34(2):283-92.

2. Pellicelli AM, Barbaro G, Palmieri F, et al. Primary pulmonary hypertension in HIV patients: a systematic review. Angiology. 2001;52(1):31-41.

3. Hsue PY, Deeks SG, Farah HH, et al. Role of HIV and human herpesvirus-8 infection in pulmonary arterial hypertension. AIDS (London, England). 2008; 22(7):825-33.

4. Waddingham PH, Bhattacharyya S, Zalen JV, Lloyd G. Contractile reserve as a predictor of prognosis in patients with non-ischaemic systolic heart failure and dilated cardiomyopathy: a systematic review and meta-analysis. Echo Res Pract. 2018:5(1):1-9.

5. Hsu S, Houston BA, Tampakakis E, et al. Right ventricular functional Reserve in Pulmonary Arterial Hypertension. Circulation. 2016;133(24):2413-22.

6. Groepenhoff H, Vonk-Noordegraaf A, van de Veerdonk MC, Boonstra A, Westerhof $\mathrm{N}$, Bogaard HJ. Prognostic relevance of changes in exercise test variables in pulmonary arterial hypertension. PLoS One. 2013;8(9):1-11.

7. Maron BA, Cockrill BA, Waxman AB, Systrom DM. The invasive cardiopulmonary exercise test. Circulation. 2013;127(10):1157-64.

8. Naeije R, Saggar R, Badesch D, et al. Exercise-Induced Pulmonary Hypertension: Translating Pathophysiological Concepts Into Clinical Practice. Chest. 2018;154(1):10.

9. Foster EL, Arnold JW, Jekic M, et al. MR-compatible treadmill for exercise stress cardiac magnetic resonance imaging. Magn Reson Med. 2012;67(3):880-9.

10. Alaiti MA, Goud A, Ramani G, et al. Design of the exercise MRI evaluation of HIV-pulmonary arterial hypertension longitudinal determinants (EXALTED) trial. J Cardiovasc Med. 2017;18:11.

11. Mahler DA, Wells CK. Evaluation of clinical methods for rating dyspnea. Chest. 1988;93(3):580-6.

12. Beaver WL, Wasserman $K$, Whipp BJ. A new method for detecting anaerobic threshold by gas exchange. J Appl Physiol (1985). 1986;60(6):2020-7.

13. Barber NJ, Ako EO, Kowalik GT, et al. Magnetic Resonance-Augmented Cardiopulmonary Exercise Testing: Comprehensively Assessing Exercise Intolerance in Children With Cardiovascular Disease. Circ Cardiovasc Imaging. 2016;9:12.

14. Kramer CM, Barkhausen J, Flamm SD, Kim RJ, Nagel E. Society for Cardiovascular Magnetic Resonance Board of Trustees Task Force on Standardized P. Standardized cardiovascular magnetic resonance (CMR) protocols 2013 update. J Cardiovasc Magn Reson. 2013;15:91

15. Lin HY, Bender JA, Ding Y, et al. Shared velocity encoding: a method to improve the temporal resolution of phase-contrast velocity measurements. Magn Reson Med. 2012;68(3):703-10.

16. Aandal G, Nadig V, Yeh V, Rajah P, Jenkins T, Sattar A, et al. Evaluation of left ventricular ejection fraction using through-time radial GRAPPA. J Cardiovasc Magn Reson. 2014;16(1):79.

17. Fletcher GF, Ades PA, Kligfield P, et al. Exercise standards for testing and training: a scientific statement from the American Heart Association. Circulation. 2013;128(8):873-934.

18. Sanz J, Kuschnir P, Rius T, et al. Pulmonary arterial hypertension: noninvasive detection with phase-contrast MR imaging. Radiology. 2007;243(1):70-9.

19. Koo TK, Li MY. A guideline of selecting and reporting Intraclass correlation coefficients for reliability research. J Chiropr Med. 2016:15(2):155-63.

20. Lafountain RA, da Silveira JS, Varghese J, et al. Cardiopulmonary exercise testing in the MRI environment. Physiol Meas. 2016;37(4):N11-25.

21. Webel AR, Perazzo J, Longenecker $C T$, et al. The influence of exercise on cardiovascular health in sedentary adults with human immunodeficiency virus. J Cardiovasc Nurs. 2018:33(3):239-47.

22. Chisati EM, Vasseljen O. Aerobic endurance in HIV-positive young adults and HIV-negative controls in Malawi. Malawi Med J. 2015;27(1):5-9.

23. Kiely DG, Elliot CA, Sabroe I, Condliffe R. Pulmonary hypertension: diagnosis and management. BMJ. 2013;346:f2028.

24. Tolle JJ, Waxman AB, Van Horn TL, Pappagianopoulos PP, Systrom DM. Exercise-induced pulmonary arterial hypertension. Circulation. 2008; 118(21):2183-9. 
25. Holloway CJ, Ntusi N, Suttie J, Mahmod M, et al. Comprehensive cardiac magnetic resonance imaging and spectroscopy reveal a high burden of myocardial disease in HIV patients. Circulation. 2013;128(8):814-22.

26. Luetkens JA, Doerner J, Schwarze-Zander C, et al. Cardiac magnetic resonance reveals signs of subclinical myocardial inflammation in asymptomatic HIVinfected patients. Circ Cardiovasc Imaging. 2016;9(3):e004091.

27. Swift AJ, Rajaram S, Capener D, et al. LGE patterns in pulmonary hypertension do not impact overall mortality. JACC Cardiovasc Imaging. 2014;7(12):1209-17.

28. Lewis GD, Shah RV, Pappagianopolas PP, Systrom DM, Semigran MJ. Determinants of ventilatory efficiency in heart failure: the role of right ventricular performance and pulmonary vascular tone. Circ Heart Fail. 2008; (4):227-33.

29. Matsumoto $\mathrm{A}$, Itoh $\mathrm{H}$, Eto $\mathrm{Y}$, et al. End-tidal $\mathrm{CO} 2$ pressure decreases during exercise in cardiac patients: association with severity of heart failure and cardiac output reserve. J Am Coll Cardiol. 2000;36(1):242-9.

30. Tanabe Y, Hosaka Y, Ito M, Ito E, Suzuki K. Significance of end-tidal P (CO(2)) response to exercise and its relation to functional capacity in patients with chronic heart failure. Chest. 2001;119(3):811-7.

31. Cicoira M, Zanolla L, Franceschini L, et al. Skeletal muscle mass independently predicts peak oxygen consumption and ventilatory response during exercise in noncachectic patients with chronic heart failure. J Am Coll Cardiol. 2001:37(8):2080-5.

32. Echeverria P, Bonjoch A, Puig J, et al. High prevalence of sarcopenia in HIVinfected individuals. Biomed Res Int. 2018;2018:5074923.

33. Webel AR, Sattar A, Funderburg NT, et al. Alcohol and dietary factors associate with gut integrity and inflammation in HIV-infected adults. HIV Med. 2017;18(6):402-11.

34. Robinson-Papp J, Simpson DM. Neuromuscular diseases associated with HIV-1 infection. Muscle Nerve. 2009;40(6):1043-53.

35. Koethe JR, Heimburger DC, PrayGod G, Filteau S. From wasting to obesity: the contribution of nutritional status to immune activation in HIV infection. J Infect Dis. 2016;214(Suppl 2):S75-82.

\section{Publisher's Note}

Springer Nature remains neutral with regard to jurisdictional claims in published maps and institutional affiliations.

Ready to submit your research? Choose BMC and benefit from:

- fast, convenient online submission

- thorough peer review by experienced researchers in your field

- rapid publication on acceptance

- support for research data, including large and complex data types

- gold Open Access which fosters wider collaboration and increased citations

- maximum visibility for your research: over $100 \mathrm{M}$ website views per year

At $\mathrm{BMC}$, research is always in progress.

Learn more biomedcentral.com/submissions 